\title{
Complicações crônicas em diabetes, estratégias e qualidade dos serviços
}

Amanda Oliveira S. M. Silveira ${ }^{1}$; Reila Silva P. Aires ${ }^{2}$; Ednólia Gomes V. Fernandes ${ }^{3}$; Mabel Duarte A. Gomides ${ }^{4}$, Michelly de Melo Alves ${ }^{5}$; Geraldo Sadoyama ${ }^{6}$

\section{Resumo}

O Diabetes Mellitus (DM) é uma doença crônica, de evolução insidiosa e progressiva, de crescente importância na saúde pública, devido aumento na sua incidência, prevalência, morbidade, mortalidade e custos econômicos. Atualmente existem 415 milhões de pessoas afetadas em todo o mundo e estima-se que $12 \%$ do orçamento global em saúde são gastos em DM. Essa enfermidade está associada com complicações microvasculares, que incluem retinopatia, neuropatia e nefropatia, e com complicações macrovasculares, que englobam doença cardiovascular e cerebrovascular, sendo essas relacionadas com uma carga socioeconômica e custos substanciais para o tratamento das mesmas. Portanto o planejamento de estratégias de educação em diabetes por equipes multidisciplinares é fundamental para o controle adequado da enfermidade e de suas complicações, sendo considerado relevante no cuidado integral ao paciente com DM. Dessa forma a avaliação da qualidade dos serviços prestados faz-se necessário para analisar a satisfação entre os clientes. Portanto serviços em saúde que ofereçam qualidade, possibilitam aos pacientes maior adesão ao tratamento, redução das complicações relacionadas com a doença, menores custos e melhor qualidade de vida.

Palavras Chave: diabetes; complicações crônicas; qualidade de serviços.

\footnotetext{
1-Médica endocrinologista, Mestranda em Gestão Organizacional - UFG - Catalão-GO amanda.osm@bol.com.br 2-Médica Intensivista, Mestranda em Gestão Organizacional - UFG- Catalão- GO reilasp@gmail.com 3-Enfermeira, Catalão-GO ednoliavarjão@yahoo.com

4-Médica dermatolgista, Mestre em Gestão Organizacional- UFG- Catalão-GO mabel@dermaclinicagoias.com.br 5-Enfermeira, Mestranda em Gestão Organizacional -UFG- Catalão-GO michellymeloa@hotmail.com 6-Biólogo, Instituto de Biotecnologia, Professor do Mestrado em Gestâo Organizacional -UFG- Catalão -GO sadoyama@ufg.br
} 


\section{Introdução}

O Diabetes Mellitus (DM) é uma doença crônica, de evolução insidiosa e progressiva, de crescente importância na saúde pública, devido aumento na sua incidência, prevalência, morbidade, mortalidade e custos econômicos.

Franco e Sartorelli (2009), apontam que em 1985, existiam 30 milhões de adultos com DM no mundo. Segundo dados da Federação Internacional de Diabetes (IDF) em 2015, atualmente há 415 milhões de pessoas com diabetes, e estimativas sugerem que esta projeção deverá aumentar para 642 milhões de indivíduos em 2040, sendo que metade desconhecem que são portadores da doença e integram o grupo de pacientes com maior risco de apresentarem complicações com maiores custos. Nota-se ainda que $12 \%$ do orçamento global em saúde são gastos com Diabetes e a cada 6 segundos uma pessoa morre decorrente da enfermidade.

De acordo com as Diretrizes da sociedade Brasileira de Diabetes (2015), esse aumento de indivíduos diabéticos é proveniente do crescimento e envelhecimento populacional, da maior urbanização, da crescente prevalência de obesidade e sedentarismo, bem como da maior sobrevida de pacientes diabéticos. Avaliar a prevalência atual e futura do DM é importante para planejar e alocar recursos de forma racional (WHO, 2002).

O Diabetes está associado com complicações microvasculares, que incluem retinopatia, neuropatia, nefropatia e com complicações macrovasculares, que englobam doença cardiovascular e cerebrovascular, sendo essas relacionadas com uma carga socioeconômica e custos substanciais para o tratamento das mesmas. Porém o custo intangível da dor, ansiedade, inconveniência e perda da qualidade de vida não são mensuráveis, mas refletem de forma relevante sobre os indivíduos afetados e suas famílias (SBD, 2015).

Evidências de estudos conduzidos anteriormente demonstram a importância do controle glicêmico adequado e sustentado entre os pacientes portadores de Diabetes Tipo 1 e Diabetes tipo 2 para redução das complicações crônicas. Esses estudos enfatizam o papel central dos níveis de hemoglobina glicosilada (HBA1c), dentro de valores considerados adequados (6,5-7,0\%), dessa forma sendo, responsáveis para motivar profissionais de saúde e pacientes para o controle constante dos níveis de glicemia.

Porém o modelo de tratamento atual do DM no Brasil tem se mostrado ineficiente. Segundo Mendes et al. (2010), um estudo multicêntrico realizado em 4 regiões do país (Nordeste, Centro-Oeste, Sudeste e Sul), com 6701 pacientes, demonstrou que somente $10,4 \%$ dos pacientes com DM1 e $26,8 \%$ dos com DM2, apresentavam uma hemoglobina glicada(HBA1c) abaixo do alvo de $7 \%$. Estes resultados provavelmente são decorrentes dos pacientes em não aderirem as intervenções não farmacológicas recomendadas, além da baixa eficácia dos medicamentos disponíveis e a não aderência a terapia medicamentosa.

Segundo Grillo et al. (2013) o cuidado ao paciente com DM inclui intervenções multidisciplinares e em todos os níveis de atenção à saúde, e o sucesso dessas depende da capacidade do paciente em assumir mudanças no estilo de vida, de manter os cuidados recomendados, e ainda de ter iniciativa, para identificar, solucionar ou buscar auxílio para os problemas que surgem no decorrer da enfermidade. $\mathrm{O}$ aprimoramento dessas capacidades é facilitado pela educação em diabetes, sendo essa, parte relevante no cuidado integral ao paciente com DM. 
Além das intervenções medicamentosas e educativas, a qualidade do atendimento prestado aos pacientes diabéticos é outro fator de relevância no tratamento. No Brasil tem sido avaliada, e vários problemas têm sido apontados. Dessa forma observa-se que quanto melhor a qualidade dos serviços ofertados, maior adesão ao tratamento, melhores resultados no controle da patologia, redução das complicações relacionadas ao diabetes e menores custos.

Existem evidências que várias intervenções, podem reduzir a incidência ou retardar a progressão das complicações crônicas, tanto no DM1 (Diabetes Control and Complications Trial - DCCT,1993) como do DM2 (UK Prospective Diabetes Study - UKPDS, 1998). Por isso, programas que visem um bom controle metabólico dos diabetes, além de melhorar a qualidade de vida dos indivíduos com a doença, têm grande impacto, de forma significativa, para redução dos custos atualmente existentes em seu tratamento.

Nesse sentido, este artigo busca rever conceitos sobre diabetes, epidemiologia e complicações crônicas relacionadas com a doença, ações estratégicas para educação em diabetes, qualidade dos serviços em saúde e escala SERVPERF.

\section{Desenvolvimento}

\subsection{Diabetes Mellitus - Conceito e Classificação}

O Diabetes Mellitus (DM) representa um grupo de distúrbios metabólicos, com etiologias diversas, caracterizados por hiperglicemia, que resulta de uma secreção deficiente de insulina, resistência periférica à ação da insulina ou ambas. A hiperglicemia crônica do diabetes está associada com disfunção ou falência de diversos órgãos, especialmente olhos, rins, nervos, coração e vasos sanguíneos (ADA, 2012).

Atualmente a classificação do Diabetes baseia-se na etiologia, segundo a organização Mundial de Saúde (OMS), Associação Americana de Diabetes (ADA) e Sociedade Brasileira de Diabetes (SBD), e inclui 4 classes clinicas: DM tipo 1 (DM1), DM tipo 2 (DM2), outros tipos específicos de DM e diabetes mellitus gestacional (DMG).

O DM2 é a forma presente em $90 \%$ a $95 \%$ dos casos e caracteriza-se por defeitos na ação e secreção da insulina. O diagnóstico, na maioria dos casos, é feito a partir dos 40 anos, embora possa ocorrer mais cedo, até mesmo na adolescência. Existem várias e diferentes causas para DM2. Esta forma de DM, em geral cursa sem o devido diagnóstico por muitos anos, uma vez que sua evolução é lenta e gradual, com sintomas inicialmente leves ou até mesmo imperceptíveis. A despeito de sua evolução lenta, esses indivíduos apresentam elevado risco para o desenvolvimento de complicações microvasculares e macrovasculares. Esse tipo de diabetes ocorre principalmente em pacientes com sobrepeso ou obesidade, histórico familiar de DM2 e de DMG, bem como nos portadores de hipertensão arterial ou dislipidemia. (ADA, 2012).

De acordo ainda com ADA (2012), o DM1 é o resultado da destruição de células betapancreáticas com consequente deficiência de insulina. Representa 5 a 10\% dos casos de diabetes. Na maioria dos casos, essa destruição de células beta é mediada por autoimunidade, porém existem casos em que não há evidências do processo autoimune sendo assim, referidos como forma idiopática de DM1. A presença de anticorpos pode surgir meses ou anos antes do diagnóstico clínico, ou seja, na fase 
pré-clínica da doença, e em até $90 \%$ dos indivíduos quando se detecta a hiperglicemia. Além do componente autoimune, o DM1 apresenta intensa associação a determinados genes do sistema leucocitário humano (HLA), alelos que podem suscitar o desenvolvimento da doença ou proteger contra ela. É mais frequente em crianças, mas pode ocorrer em adultos, sendo identificada neste caso como diabetes autoimune latente do adulto (LADA).

Outros tipos específicos de DM incluem formas menos comuns, cujos defeitos ou processos causadores podem ser identificados. Estão incluídos nesse grupo, os defeitos genéticos na função de célula beta, defeitos genéticos na ação da insulina, doenças do pâncreas exócrino, endocrinopatias, DM provocado por medicamentos, agentes químicos, ou infecções, formas incomuns de DM autoimune e síndromes genéticas raras. O DM gestacional refere-se as alterações de intolerância à glicose com diagnóstico ou início durante a gestação. (ADA, 2012).

\subsection{Epidemiologia}

Conforme já descrito, uma epidemia de diabetes está em curso. No Brasil segundo dados de IDF (2015), são 14,3 milhões de pessoas afetadas entre 20 a 79 anos de idade, com estimativas para alcançarem 23,2 milhões de indivíduos nos próximos 25 anos. Observa-se ainda a ocorrência de 30900 indivíduos com DM1 na faixa etária de 0 a 14 anos.

Segundo Malerbi et al. (1992), um estudo multicêntrico sobre a prevalência de diabetes no Brasil, realizado em 9 capitais brasileiras, com a população de 30 a 69 anos, é um marco importante nessa área de pesquisa e no redimensionamento dessa doença. Seus resultados possibilitaram o reconhecimento da importância do diabetes como grave problema de saúde no Brasil, onde estimou-se a prevalência em 7,6\%, com taxas mais elevadas sendo encontradas nas cidades das regiões Sul e Sudeste do Brasil. Dados mais recentes apontam para as taxas mais elevadas, como $13,5 \%$ em São Carlos (BOSI et al., 2009), 12,1 \% no estudo de Ribeirão Preto (MORAES et al, 2010) e $20 \%$ em um estudo realizado em 6 capitais brasileiras, com servidores de universidade públicas, sendo que aproximadamente metade dos casos sem diagnóstico prévio (MENDES et al., 2010).

Nota-se ainda diferenças no predomínio de diversos países e grupos étnicos, sendo descritos taxas mais elevadas para Nauru, na Oceania, e para os índios Pima, no Arizona, EUA, onde metade da população adulta apresenta DM (EKOÉ JM et al., 2008). No Brasil observou-se elevada prevalência entre os índios Xavante: de 28,2\% em ambos os sexos, de $18,4 \%$ em homens e de $40,6 \%$ em mulheres. (DAL FRABBRO et al., 2008).

A incidência de DM2 em grandes populações é difícil de ser avaliada pois requer seguimento durante anos e medições seriadas de glicemias. Já para os pacientes portadores de DM1 sua incidência tem mostrado acentuada variação geográfica, apresentando taxas por 100 mil indivíduos afetados com menos de 15 anos de idade: 38,4 na Finlândia, 7,6 no Brasil e 0,5 na Coréia (ONKAMO, et al., 1999). Recentemente observou-se aumento na incidência de DM1, principalmente na população infantil com menos de 5 anos de idade. (KARVONEN et al., 2000).

Os dados de mortalidade obtidos, mediante atestado de óbito, subestimam a importância do diabetes, pois é frequente essa doença não ser mencionada na declaração de óbito, em especial dos idosos, nos quais estão presentes, simultaneamente, várias doenças crônicas. Devido as suas complicações, particularmente as cardiovasculares e cerebrovasculares que figuram como a causa 
de óbito nesses pacientes, frequentemente o diabetes é omitido, uma vez que essas complicações estão entres as causas citadas nas estatísticas de mortalidade.

O diabetes tem se tornado uma das principais causas de morte, certamente há notificação inadequada do número de mortes atribuídas ao diabetes, por se considerar essa doença como causa secundária de óbitos registrados. Estudos brasileiros sobre mortalidade por DM, mostram que quando se analisa a mortalidade pelo método de causas múltiplas, ou seja quando se descreve DM na declaração de óbito, demonstram que a mortalidade por DM aumenta até 6,4 vezes (FRANCO, 2004).

\subsection{Complicações Crônicas}

A alta morbidade e mortalidade associadas ao DM, tanto do DM1 como do DM2, é consequência das complicações crônicas que correspondem lesões microvasculares (retinopatia, nefropatia, neuropatia) e macrovasculares (doença cardiovascular e cerebrovascular).

A doença macrovascular no paciente diabético situa-se entre as principais complicações crônicas dos portadores de DM2, e corresponde a mesma doença aterosclerótica que incide em uma população não diabética, porém ocorre de forma mais precoce, mais frequente e mais grave. Os fatores de risco modificáveis das doenças cardiovasculares são a hiperglicemia, hipertensão arterial, dislipidemia, microalbuminúria, tabagismo e sedentarismo e estes estão relacionados com a prevenção da mesma (GAEDE et al., 2008, STELLERR, 2006).

Segundo Klein e Klein (2000), a retinopatia diabética (RD) é principal causa de cegueira em pessoas com 20 a 74 anos. Após 20 anos de evolução do DM, 60 \% dos indivíduos diabéticos tipo 2 e mais $90 \%$ dos portadores de DM1 poderão apresentar algum grau de retinopatia.

O estudo norte-americano Diabetes Control and Complications Trial (DCCT,1993) comparou a terapia insulínica intensiva à insulinoterapia convencional em pacientes com DM1. Os pacientes submetidos ao controle intensivo apresentaram redução de $76 \%$ no risco de desenvolvimento de retinopatia. Em pacientes com Diabetes tipo 2 (DM2), o estudo United Kingdom Prospective Diabetes Study (UKPDS,1998) demonstrou a importância do controle intensivo da pressão arterial. Após nove anos de acompanhamento e controle pressórico diminuiu-se o risco de progressão da retinopatia em $47 \%$. O risco de cegueira pela retinopatia RD pode ser reduzido a $5 \%$ quando o diagnóstico é feito em tempo adequado e o tratamento realizado corretamente, antes que as alterações irreversíveis possam se instalar. (FERRIS, 1993)

Segundo Park (2014) a doença renal do diabetes (DRD) atualmente é classificada baseada na taxa de filtração glomerular (TFG) e/ou excreção urinária de albumina (EUA). Para TENG et al (2012) observou-se que 30 a $50 \%$ dos pacientes apresentou aumento da EUA e em aproximadamente 1/5 dos casos houve redução isolada da taxa de filtração glomerular (DWYER,2012). É a principal causa de insuficiência renal crônica (IRC) em pacientes que ingressam em programas de diálise, inclusive no Brasil.

A DRD está associada a importante aumento de mortalidade, principalmente relacionada à doença cardiovascular. De fato, o aumento da EUA é um importante fator de risco para eventos coronarianos, além de ser também um fator de risco para o desenvolvimento e progressão da DRD. Em estudos realizados no sul do Brasil, 
observou-se acometimento renal em 37\% dos pacientes com Dm2 e 34\% dos pacientes com DM1 (RODRIGUES et al., 2010).

$O$ rastreamento da DRD deve ser iniciado logo ao diagnóstico do diabetes nos pacientes com DM2 e após 5 anos do início do DM1. No entanto, pacientes com DM1, que se encontrem na puberdade ou com diabetes persistentemente descompensado devem ser rastreados antes. $O$ rastreamento deve ser anual e deve basear-se na medida da albuminúria e na estimativa da TFG (ADA, 2013).

Conforme descreve Boulton et al (1998), a neuropatia diabética é uma complicação crônica definida pela presença de sintomas e/ou sinais de disfunção dos nervos periféricos em indivíduos com DM após exclusão de outras causas. É uma complicação desabilitante, que piora a qualidade de vida do paciente e, infelizmente, muitas vezes é subdiagnosticada e subtratada. O bom controle metabólico reduz o surgimento da neuropatia e sua gravidade. (DCCT, 1993).

As formas mais comuns são as polineuropatias simétricas generalizadas, sendo a mais frequente de todas as formas, seguindo-se as autonômicas (cardiovascular, respiratória, digestiva e gênito-urinária) e mais raramente a sensorial aguda, enquanto as formas focais e multifocais são menos frequentes. Afeta $50 \%$ dos portadores de DM2 e em menor proporção os DM1. Em qualquer dos tipos, a exposição crônica à hiperglicemia constitui-se o fator etiológico comum, conforme demostrado no DCCT (1993) e no UKPDS (1998).

Nesse sentido, o diagnóstico da neuropatia diabética é essencialmente clínico, através da anamnese (queixas de dormência ou queimação em membros inferiores, principalmente; embora os membros superiores podem ser afetados também, formigamentos, pontadas, choques, agulhadas, desconforto ou dor ao toque dos lençóis e cobertores) e da avaliação da sensibilidade (tátil, térmica, vibratória e dolorosa), pesquisa de reflexos profundos e de hipotensão postural.

De acordo com Bakker et al. (2015), o pé diabético é conceituado no glossário do Guidance (Recomendações), do IWGDF (International Working Group on the Foot ou Grupo de Trabalho Internacional sobre Pé Diabético) como infecção, ulceração e/ou destruição de tecidos moles associados a alterações neurológicas e vários graus de doença arterial periférica (DAP) nos membros inferiores. Constitui-se em uma das complicações mais nocivas, uma vez que as ulcerações podem levar a amputações maiores ou menores. Segundo Boulton et al (2005) a incidência anual situa-se entre $2 \%$ e $4 \%$ e a prevalência documentada varia de 4 a $10 \%$, sendo mais elevada em países com baixa situação econômica. A incidência cumulativa de ulceração ao longo da vida entre pacientes diabéticos situa-se entre $25 \%$, ressaltando que $85 \%$ das úlceras precedem as amputações.

O aspecto mutilador da complicação é um problema de saúde relevante pelo impacto socioeconômico global resultante: a cada minuto ocorrem três amputações decorrentes do DM, segundo IWGDF (2015). Sua presença implica em elevada mortalidade, piora na qualidade de vida, absenteísmo e aposentadoria precoce.

O treinamento de profissionais de saúde, inclusive de médicos, é crucial para a aplicação de técnicas para rastreamento e diagnóstico de polineuropatia diabética e doença arterial periférica. Sua finalidade é identificar os pacientes em risco de ulceração (BAKKER et al, 2015, PEDROSA et al 2004,). Análises da Suécia mostraram que a prevenção intensiva (educação do paciente, uso de calçados adequados e acesso a cuidados regulares pela equipe multiprofissional) destinada a pacientes com risco elevado de ulceração é efetiva se a incidência de úlcera e amputação é reduzida em 25 \% (RAGNARSON et al., 2004). 
O Consenso Internacional do IWGDF recomenda a implantação de serviços básicos na comunidade, de ambulatórios ligados a hospitais ou centros especializados, de modo a se estabelecer, gradualmente uma rede integrada para atendimento aos portadores de DM com graus vários de problemas nos pés.

\subsection{Ações Estratégicas}

A educação é uma ferramenta importante no tratamento do paciente com DM, e segundo a Associação Americana de Diabetes, todos os pacientes com DM deveriam receber educação para o autocuidado. Decorrente disso , em 2006 foi desenvolvido a National Standards for Diabetes Self-Management Education (DSME), e revisadas a cada 5 anos, com o objetivo de garantir a qualidade da educação para 0 autocuidado ofertada aos pacientes com DM, nos mais diversos cenários e tem como principais objetivos a preparação do paciente na tomada de decisões referentes ao seu tratamento, estimulando o comportamento direcionado para o autocuidado, o que propiciaria resolução dos problemas com a participação ativa da equipe de saúde. (FUNNELL et al, 2010).

A Associação Americana de Educadores em Diabetes (AADE) elaborou regras, entre elas que os pacientes com DM sejam assistidos por uma equipe multidisciplinar formada por médicos, educadores físicos, nutricionistas, enfermeiros, psicólogos, assistentes sociais, farmacêuticos, dentistas. Determinou-se a criação de projetos que visam encorajar os pacientes na modificação do estilo de vida e na adoção de comportamentos de autocuidado com informações básicas sobre os diabetes, treinamento dos pacientes, familiares e cuidadores no uso de dispositivos disponíveis no tratamento. Essas estratégias para o autocuidado podem ser realizadas através de entrevistas individuais ou em pequenos grupos (dinâmicas de grupos) e através de encontros educativos.

A AADE (2002) propõe a aplicação de 7 medidas de comportamentos relacionados a avaliação comportamental para reconhecer a qualidade dos resultados decorrentes de processo educativo efetivo, são elas:

- Comer saudavelmente: orientação para leitura de rótulos, planejamento, preparo e fracionamento de refeições, controle das porções e contagem de carboidratos dos alimentos

- Praticar atividade física: desenvolvimento de um plano de atividades equilibradas e adequadas ao plano alimentar e de medicação

- Vigiar as taxas: orientações sobre escolha de equipamentos, diário glicêmico, frequência dos testes, valores-alvo, interpretação e utilização de resultados

- Tomar os medicamentos: orientação da equipe da saúde sobre os benefícios dos medicamentos, técnicas de aplicação, ao transporte e manuseio de insulina

- Adaptar saudavelmente: identificar a motivação do indivíduo para mudança de comportamento, auxiliando-o a estabelecer metas clínicas e comportamentos alcançáveis

- Resolver problemas: os educadores devem auxiliar a resolver problemas relacionados com hiperglicemias e hipoglicemias, além de ajudar a lidar com barreiras físicas, emocionais, cognitivas e financeiras, incentivando a criação de estratégias para enfrentamento.

- Reduzir risco: orientar sobre os objetivos terapêuticos e cuidados preventivos para diminuir riscos de complicações como incentivo a parar de fumar, inspecionar pés, monitorar a pressão arterial, automonitorização da glicemia e manutenção de registros pessoais. 
A educação em DM no Brasil acontece através de iniciativas pontuais, desde serviços ligados ao setor público (estaduais e municipais), entidade privadas (hospitais e laboratórios) ou organizações não governamentais (associações de pacientes). Em 2007 a SBD mais Associação de Diabéticos Juvenil (ADJ) e IDF iniciaram o programa de Educando Educadores - Curso de Qualificação de profissionais de Saúde em Educação em Diabetes que envolve os sete comportamentos do autocuidado definidos pela AAEE (SBD,2015).

Em parceria com o Conselho Federal de Farmácia, ADJ, e apoio da IDF, criouse o programa Apriframa, aprimoramento em diabetes para farmacêuticos, curso ministrado aos farmacêuticos de todo o Brasil. A ANVISA regulamentou RDC 44/2009 onde normatizou-se a prestação de serviços farmacêuticos, entre eles a execução da glicemia capilar (BRASIL, 2009).

\subsection{Qualidade dos serviços de saúde}

De acordo com Gama \& Saturno (2013) um aspecto fundamental no conceito de qualidade em saúde, refere-se que o entendimento desta, não depende de um único fator, mas sim, da existência de componentes, atributos e dimensões. Um grupo de dimensões muito utilizado e que serviu de base para a construção de indicadores de qualidade em várias partes do mundo foi o do Instituto de Medicina dos Estados Unidos (IOM), que posteriormente foi adaptado pela Organização Mundial de Saúde (OMS), composto pelas 6 dimensões sumarizadas no quadro abaixo:

Quadro 1- Dimensões da qualidade dos serviços em saúde

\begin{tabular}{|l|l|}
\hline Segurança & $\begin{array}{l}\text { Ausência de lesões devido à assistência à saúde que supostamente deve } \\
\text { ser benéfica. } \\
\text { Sistemas de saúde seguros diminuem risco de danos aos pacientes. }\end{array}$ \\
\hline Efetividade & $\begin{array}{l}\text { Prestação de serviços baseados no conhecimento científico a todos os } \\
\text { que podem beneficiar-se destes, e evitar prestar serviços aqueles que } \\
\text { provavelmente não se beneficiarão (evitar a infra e supra utilização, } \\
\text { respectivamente). }\end{array}$ \\
\hline $\begin{array}{l}\text { Atenção centrada no } \\
\text { paciente }\end{array}$ & $\begin{array}{l}\text { Envolve o respeitar o paciente, considerando suas preferências } \\
\text { individuais, necessidades assegurando que a tomada de decisão clínica } \\
\text { depende desses valores. }\end{array}$ \\
\hline Oportunidade/Acesso & $\begin{array}{l}\text { Redução de esperas e atrasos, às vezes prejudiciais, tanto para os que } \\
\text { recebem como para os que prestam a assistência à saúde. }\end{array}$ \\
\hline Eficiência & $\begin{array}{l}\text { Prevenção de desperdício de equipamentos, suprimentos, ideias e } \\
\text { energias. }\end{array}$ \\
\hline Equidade & $\begin{array}{l}\text { Prestação de serviços que não variam a qualidade segundo } \\
\text { características pessoais, tais como gênero, etnia, localização geográfica } \\
\text { e status socioeconômico. }\end{array}$ \\
\hline FONTE: GAMA \& SATURNO \\
(2013)
\end{tabular}

Portanto é crescente o interesse em mensurar a qualidade dos serviços prestados, sendo assim vários pesquisadores tem buscado aprimorar técnicas de medição, e vários instrumentos tem sido propostos para avaliação, todavia destacase os instrumentos SERVQUAL e SERVPERF (MIGUEL \& SALOMI, 2004).

\subsection{Escala SERVPERF}

A escala SERVPERF é um instrumento proposto por Cronin \& Taylor (1992) para avaliar a qualidade dos serviços internos em relação a satisfação do usuário. 
Este instrumento foi extraído do instrumento SERVQUAL proposto no trabalho de Parasuraman et al.(1988), cujo modelo serve para avaliação da qualidade em serviços, porém avalia a diferença entre a expectativa e a percepção que os clientes têm do serviço ofertado, e tem como base as cinco dimensões: Tangibilidade, Credibilidade, Presteza, Segurança e Empatia. Esse modelo é composto por 44 itens que compõem as cinco dimensões da qualidade citadas, sendo esse modelo realizado em duas etapas. Na primeira são mensuradas as expectativas dos clientes, e na segunda, são mensuradas as percepções dos clientes em relação ao serviço prestado. Na figura abaixo observa-se a evolução das dimensões, inicialmente avaliadas em dez, e depois condensadas em cinco.

Quadro 2 - Evolução nas dimensões da qualidade

\begin{tabular}{|c|c|c|c|c|c|}
\hline \multicolumn{6}{|c|}{ DIMENSÕES SERVQUAL } \\
\hline $\begin{array}{c}\text { Dez dimensões } \\
\text { originais }\end{array}$ & $\begin{array}{l}\text { Aspectos } \\
\text { Tangíveis }\end{array}$ & Confiabilidade & Presteza & Segurança & Empatia \\
\hline $\begin{array}{l}\text { Aspectos } \\
\text { Tangíveis }\end{array}$ & $\begin{array}{c}\text { Questões } \\
1 \text { a } 4\end{array}$ & & & & \\
\hline Confiabilidade & & Questões 5 a 9 & & & \\
\hline Presteza & & & Questões 10 a13 & & \\
\hline Competência & & & & $\begin{array}{c}\text { Questões } \\
14 \text { a } 17\end{array}$ & \\
\hline Cortesia & & & & & \\
\hline Credibilidade & & & & & \\
\hline Segurança & & & & & \\
\hline Acessibilidade & & & & & \\
\hline Comunicação & & & & & \\
\hline $\begin{array}{l}\text { Compreensão/ } \\
\text { Conhecimento }\end{array}$ & & & & & $\begin{array}{c}\text { Questões } \\
18 \text { a } 22\end{array}$ \\
\hline
\end{tabular}

(PARASURAMAN et al., 1988)

Portanto a segunda etapa do instrumento SERVQUAL corresponde a escala SERVPERF, composta por 22 itens, proposta de uma avaliação mais direta da qualidade do serviço, e cujo modelo mede apenas as percepções dos clientes sobre os serviços prestados pela organização, não há expectativas, como na SERVQUAL.

Para Cronin e Taylor (1992) a escala SERVPERF produziu melhores resultados, as estimativas foram mais confiáveis, mais convergentes e de validade discriminante em relação a SERVQUAL, uma vez que reduziu pela metade o número de itens a serem medidos, quando se analisa apenas as percepções e conclui-se que a qualidade dos serviços prestados é mais bem avaliada apenas pelas percepções que os consumidores têm em relação ao desempenho do serviço. Segue abaixo o quadro com as questões propostas para avaliação: 
Quadro 3- Questionário de Importância dos Itens

\begin{tabular}{|c|c|}
\hline Item & Importância \\
\hline 1 & Equipamentos Modernos \\
\hline 2 & Instalações físicas são virtualmente atrativas \\
\hline 3 & Os empregados são bem vestidos e asseados \\
\hline 4 & A aparência das instalações físicas é conservada de acordo com o serviço oferecido. \\
\hline 5 & Quando algo é prometido em um certo tempo é cumprido \\
\hline 6 & Quando há algum problema com a empresa, ela é solidária e o deixa seguro \\
\hline 7 & Tem confiabilidade \\
\hline 8 & Fornece o serviço no tempo prometido \\
\hline 9 & Mantém seus registros de forma correta \\
\hline 10 & Informa exatamente quando os serviços serão executados. \\
\hline 11 & Recebe serviço imediato \\
\hline 12 & Empregados estão sempre dispostos a ajudar os clientes \\
\hline 13 & Empregados não estão sempre ocupados para responder aos pedidos dos clientes \\
\hline 14 & Empregados são confiáveis \\
\hline 15 & O sentimento de se sentir seguro em negociar com os empregados da empresa \\
\hline 16 & Empregados são educados \\
\hline 17 & Suporte adequado da empresa para cumprir suas tarefas corretamente \\
\hline 18 & Atenção Individual \\
\hline 19 & Empregados dão a você atenção pessoal \\
\hline 20 & Empregados sabem das suas necessidades \\
\hline 21 & Os objetivos da empresa são os seus objetivos \\
\hline 22 & Horário de trabalho conveniente. \\
\hline
\end{tabular}

Fonte: (Cronin \&Taylor, 1992)

\section{CONSIDERAÇÕES FINAIS}

Diante de uma epidemia de Diabetes e consequentes complicações devastadoras, com alto impacto social e econômico, torna-se fundamental o desenvolvimento de estratégias para redução dessas complicações e consequentemente dos custos relacionados com o seu tratamento.

A educação em Diabetes é a principal ferramenta que o paciente diabético disponibiliza para o autocuidado, o qual possibilitará o controle adequado da enfermidade. Para que isso aconteça é fundamental o trabalho de uma equipe multidisciplinar e qualificação constante da mesma. Além do comprometimento do paciente, torna-se necessário o envolvimento de familiares, da sociedade, entidades governamentais e políticas para conter o avanço de pessoas afetadas pela doença.

Dessa forma obter qualidade dos serviços em saúde, de forma eficiente e igualitária, principalmente no setor público, é um grande desafio. Para isso é de suma importância, avaliar a satisfação dos clientes, e para que isso ocorra é necessário levar em consideração a opinião de usuários dos serviços e funcionários. Entre os vários instrumentos utilizados para mensurar a qualidade, destaca-se a escala SERVPERF pela sua objetividade e precisão. Portanto serviços que buscam qualidade em saúde, possibilitam aos pacientes maior adesão ao tratamento, menos complicações e melhor qualidade de vida. 


\title{
Chronic complications in diabetes, strategies and quality of services
}

\begin{abstract}
Diabetes Mellitus (DM) is a chronic, insidious and progressive evolution of increasing importance in public health, due to increase in its incidence, prevalence, morbidity, mortality and economic costs. Currently there are 415 million people affected worldwide and it is estimated that $12 \%$ of the global health budget is spent on DM. This disease is associated with microvascular complications including retinopathy, neuropathy and nephropathy, and macrovascular complications, which include cardiovascular and cerebrovascular disease, and those associated with a socio-economic burden and substantial costs for treating the same. So planning education strategies in diabetes by multidisciplinary teams is essential for proper control of the disease and its complications, is considered relevant to the comprehensive care of patients with DM. Thus the evaluation of the quality of services it is necessary to analyze the satisfaction among customers. Therefore health services that offer quality, enable greater patient adherence to treatment, reduction of complications related to the disease, lower costs and better quality of life.
\end{abstract}

Keywords: diabetes; chronic complications; quality of servisse

\section{Referências}

ADA - American Diabetes Association. Diagnosis and classification of diabetes mellitus. Diabetes Care. 2012; 35 (Suppl. 1): S64-71.

ADA - American Diabetes Association. Diagnosis and classification of diabetes mellitus. Diabetes Care. 2013; 36 (Suppl. 1): S67-74.

American Association of Diabetes Educators (AADE). Disponivel em 10-08-16:< http:// www.diabeteseducator.org/professional Resources/AADE7>

American Association of Diabetes Educators (AADE). Individualization of diabetes self-management education. Diabetes Educ. 2002; 28: 741-9.

BAKKER K.; APELQVIST, J.; LIPSKY, B.A.; VAN NETTEN, J. J.;SCHAPER, N. C. The 2015 Guidance on prevention and management of foot problems in diabetes: development of an evidence-based global consensus. International Working Group on the Diabetic Foot (IWGDF). Disponivel em: <http://www.iwgdf.org>. Acesso em: 7/09/2016.

BOULTON, A.J.M.; VILEIKYTE, L.; RAGNARSON-TENNVALL, G.; APELQVIST,J. The global burden of diabetic foot disease. Lancet. 2005; 366:1719-24. 
BOULTON, A. J. M.; GRIES, F.A.; JERVELL, J. A.; Guidelines for the diagnosis and outpatient management of diabetic peripheral neuropathy. Diabetic Medicine. June 1998; Vol. 15(6): p. 508-514.

BOSI,P.L.;CARVALHO, A. M.; CONTRERA, D.;CASCALE, G.;PEREIRA, M. A.; GRONNER, M. F.;DIOGO, T. M.;TORQUATO, M. T. C. G.; OISHI, J.; LEAL, A.M. Prevalência de Diabetes melito e tolerância à glicose diminuída na população urbana de 30 a 79 anos da cidade de São Carlos, São Paulo. Arq. Brasileiros Endocrinol. Metab. 2009:53:726-732.

BRASIL - Resolução Federal RDC n0 442009 - Vigilância Sanitária. Disponível em: www.vigilanciasanitaria.sc.gov.br/

CRONIN, J.J. \& TAYLOR, S. A. Measuring service quality: a reexamination and extension. Journal of Marketing, v. 56 (3), July, p. 55-68, 1992.

DAL FABBRO, A. L.; FRANCO, L.J., DA SILVA, A.; SARTORELLI, D.S.; SOARES, L.P., FRANCO, L.F.; KUHN, P.C.; MOISÉS, S.R.; VIEIRA-FILHO, J.P.B. High prevalence of type 2 diabetes mellitus in Xavante Indians from Mato Grosso. Ethn Dis. 2013; 24(1):35-40.

DCCT. The Diabetes Control and Complications Trial Research Group. The effect of intensive treatment of diabetes on the development and progression of longterm complications in insulin-dependent diabetes mellitus. $\mathrm{N}$ Engl $\mathrm{J}$ Med. 1993;329:977-86.

DWYER, J. P; PARVING, H. H.; HUNSICKER,L.G.; Renal dysfunction in the presence of normoalbuminuria in type 2 diabetes: results from the DEMAND study. Cardiorenal Med. 2012; 2 (1): 1-10.

GAEDE P.; LUND-ANDERSEN H.; PAR VING, H-H.; Oluf , P., O. Effect of a multifactorial intervention on mortality in type 2 diabetes. $\mathrm{N}$ Engl $\mathrm{J}$ Med. 2008; 358: 580-91.

GRILLO, M. F. F.; NEUMAN, C. R.; SCAIN, S. F.; ROZENO, R. F.; GROSS, J. L.; LEITÃO, C. B. Efeito de diferentes modalidades de educação para o autocuidado a pacientes com diabetes. Ver Assoc Med Bras. 2013;59(4):400-405.

EKOÉ J. M.; REWERS,M.; WILILIAMS,R.; ZIMMET,P. The epidemiology of diabetes mellitus. 2nd ed. Oxford: Wiley-Blackwell, 2008.

FERRIS, F. L. III. How effective are tratments for diabetic retinopathy? 2008; J Am Med Assoc. 1993; 269: 1290-1.

FRANCO, Laércio Joel; SARTORELLI, Daniela Saes. Diabetes Melito: aspectos epidemiológicos. In.: Programa de Atualização em Endocrinologia e Metabologia. Porto Alegre: Artmed/Panamericanda Editora, 2009. p. 75-99.

FRANCO, Laércio Joel. Diabetes: aspectos epidemiológicos. In.: Diabetes Mellitus e Doenças Cardiovasculares. São Paulo: AC Farmacêutica, 2014. p. 12-23 
FUNELL M.M.; BROWN, T. L.; CHILDS B. P.; HAAS L. B.; HOSEY, G. M. et al. National Standards for diabetes self-management education. Diabetes Care. 2010;33 Suppl 1: S89-96.

GAEDE P.; LUND-ANDERSEN H.; PAR VING, H-H.; Oluf , P., O. Effect of a multifactorial intervention on mortality in type 2 diabetes. $\mathrm{N}$ Engl J Med. 2008; 358: 580-91.

GAMA, Z. A. S.; SATURNO, P. J.; A segurança do paciente inserida na gestão da qualidade dos serviços de saúde. Manual da ANVISA . 2013; Cap 3, p.29- 40.

GRILLO, M. F. F.; NEUMAN, C. R.; SCAIN, S. F.; ROZENO, R. F.; GROSS, J. L.; LEITÃO, C. B. Efeito de diferentes modalidades de educação para o autocuidado a pacientes com diabetes. Ver Assoc Med Bras. 2013;59(4):400-405.

IDF - International Diabetes Federation. IDF Diabetes Atlas. 7ed.,2015. Disponível em http://www.diabetesatlas.org Acesso em 14-8-16.

KARVONEN, M.;VIIK-KAJANDER, M.;MOTCHANOVA, E.; LIBMAN, I.; LAPORTE, R.; TUOMILEHTO, J. Incidence of the childhood type 1 diabetes worldwide. Diabetes Care, 2000;23: 1516-26.

KLEIN, R.; KLEIN B. E. K, Epidemiology of eye disease in diabetes, In: Flynn Jr H. W.; Smidy W. E. , editors. Diabetes and ocular disease: past, presente, and future theraphies. The Foundation of the American Academy of Ophthalmology. 2000; 19-61.

MALERBI, D; FRANCO, L. J. Multicenter study of the prevalence of diabetes mellitus and impaired glucose tolerance in the urban Brazilian population aged 30-69 yr. The Brasilian Cooperative Group on the Study of Diabetes Prevalence. Diabetes Care. 1992 Nov; 15(11): Diabetes Care. 1992;15:1509-16.

MENDES, A. B. V.; FITTIPALDI, J.A.S.; NEVES, R. C. S.; CHACRA, A. R.; MOREIRA $\mathrm{Jr}$, E.D. Prevalence and correlates of inadequate glycaemic control: results from a Nationwide survey in 6,671 adults with diabetes in Brazil. Acta Diabetol (2010) 47:137-145.

MIGUEL, P. A. C.; SALOMI, P. G. Uma revisão dos modelos para medição da qualidade em serviços. Revista Produção v. 14 no. 12004.

MORAES, Suzana Alaves de, et al. Prevalência de diabetes mellitus e identificação de fatores associados em adultos residentes em área urbana de Ribeirão Preto, São Paulo, Brasil, 2006: Projeto OBEDIARP. Cad. Saúde Pública, Rio de Janeiro, 26(5); 929-941, maio 2010.

ONKAMO P, VAANANCN S, KARVONEN M; TUOMILEHTO J. World increase in incidence of the data on published incidence trends. Diabetologia, 1999; 42(12): 1395-1403.

PARASURAMAN, A.; BERRY, L. L.; ZEITHAML, V.A., SERVQUAL: a multiple-item scale for measuring consumer perceptions of service quality". Journal of Retailing, v. 64 p. 12-37 (Spring 1988) 
PARK, C. W. Diabetic Kidney disease: from epidemiology to clinical perspectives. Diabetes Metab J. 2014; 38(4):252-60

PEDROSA, HC; ANDRADE, A.(trads), Consenso Internacional sobre Pé Diabético. Versão Brasileira; 2001. SES-DF e Ministério da Saúde; versão 2003, 2007, 2009. 2011: disponível em www.idf.org/bookshop. Acesso: 20/09/2015.

PEDROSA, H.C.; LEME, L. A. P.; NOVAES, C.; SAIGG, M.; SENA, F.; GOMES, E.B.; et al. The diabetic foot in South America: progress with the Brazilian Save the Diabetic Foot Project. Int Diabetes Monitor. 2004; 16(4):17-24.

PEDROSA, HC; PIMAZONI, A. ( trads). Guia de Bolso para Exame dos Pés 2010. Disponível wm < www.diabetes.org.br > . Acesso: 20/09/2015.

RAGNARSON T, APELQVIST J. Health economic consequences of diabetic foot lesions. Clin Infect Dis. 2004; 39(Suppl 2):S132-S9.

RODRIGUES,T. C.; PECIS, M.; CANANI, L.H. et al. Characterization of patientes with type 1 diabetes mellitus in southern Brazil: chronic complications and associated factors. Ver Assoc Med Bras. 2010; 56(1): 67-73.

Brasil, Resolução Federal RDC no 442009 - Vigilância Sanitária. Disponível em : www.vigilanciasanitaria.sc.gov.br/ Acesso em17-09-16

SBD- Sociedade Brasileira de Diabetes. Diretrizes da Sociedade Brasileira de Diabetes. São Paulo: AC Farmacêutica, 2015-2016.

Standards of medical care in diabetes-2015: summary of revisions. Diabetes Care. 2015;38(Suppl): S4-S88.

STETTLER C.; ALLEMANN, S.; JÜNI, P.; STETTLER, C.; CULL, C. A.; HOLMAN, R. R.; EGGER, M. Glycemic control and macrovasculardisease in types 1 and 2 diabetes mellitus: Meta-analysis of randomized trials. Am Heart J. 2006; 152: 2738.

TENG, J.; DWYER, K. M.; HILL,P..; SEE,E.; EKINCI,E.I.; JERUMS,G.; MACISAAC, R.J. Spectrum of renal disease in diabetes. NEPHROLOGY. 2014; vol (19)528-536.

UKPDS-UK Prospective Diabetes Study Group. Effect of Intensive blood glucose control with sulfphonyureas or insulin compared with conventional treatment and risk of complications in pacientes with type 2 diabetes. Lancet. 1998;352:83753.

World Health Organization. The World Health Organization report 2002: reducing risks, promoting healthy life. Geneve: WHO, 2002. 
